\title{
Diallel Cross Analysis for some White Maize Inbred Lines under Two Nitrogen Levels
}

\author{
M. M. Kamara ${ }^{1}$; S. A. Okasha ${ }^{* 2}$ and Kh. A. M. Ibrahim ${ }^{3}$ \\ ${ }^{1}$ Agronomy Dept., Faculty of Agric., Kafrelsheikh University, Egypt \\ ${ }^{2}$ Agronomy Dept., Faculty of Agric., Suez Canal University, Egypt \\ ${ }^{3}$ Agronomy Dept., Faculty of Agric., The New Valley University, Egypt
}

Received: $20 / 03 / 2019$

\begin{abstract}
A half diallel cross among seven white inbred lines of maize was made in 2016 growing season. The resulted of $28 \mathrm{~F}_{1}$ crosses along with the check hybrid SC130 were evaluated under two different nitrogen levels, i.e., 90 and 120 $\mathrm{kg} \mathrm{N} /$ fed using a randomized complete block design (RCBD) with three replications at the Experimental Farm, Faculty of Agriculture, Kafrelsheikh University in 2017 growing season, to estimate general (GCA) and specific (SCA) combining ability effects and their interactions with nitrogen levels as well as identify type of gene action controlling the inheritance of the studied traits. Data were recorded on days to $50 \%$ silking, plant height, ear height, ear length, ear diameter, No. of rows/ear, No. of kernels/row and grain yield ( $\operatorname{ard} / \mathrm{fed})$. The results showed that, the mean squares due to nitrogen levels (D), genotypes (G), crosses $(\mathrm{Cr}$.), $\mathrm{G} \times \mathrm{N}$ and $\mathrm{Cr} . \times \mathrm{N}$ interactions were significant for all the studied traits. General (GCA) and specific (SCA) combining ability mean squares were highly significant for all the studied traits under both and across nitrogen levels. Both GCA and SCA effects were significantly interacted with nitrogen levels for all the studied traits, except GCA $\times \mathrm{N}$ for ear diameter. The non-additive gene action played an important role in the inheritance of all the studied traits under the two nitrogen levels and the combined data, except days to $50 \%$ silking under $\mathrm{N} 1$ level. The inbred lines $\mathrm{P}_{1}$ and $\mathrm{P}_{7}$ showed the best desirable GCA effects for earliness and $\mathrm{P}_{2}$ and $\mathrm{P}_{6}$ for shorter plants and lower ear placement. Whereas, $\mathrm{P}_{2}, \mathrm{P}_{3}$ and $\mathrm{P}_{4}$ were the best general combiners for grain yield. The crosses $\mathrm{P}_{1} \times \mathrm{P}_{3}, \mathrm{P}_{2} \times \mathrm{P}_{7}, \mathrm{P}_{3} \times \mathrm{P}_{6}, \mathrm{P}_{4} \times \mathrm{P}_{5}$ and $\mathrm{P}_{5} \times \mathrm{P}_{7}$ had the best SCA effects for grain yield as well as one or more of its components under both and across nitrogen levels. The two crosses $\mathrm{P}_{2} \times \mathrm{P}_{7}$ and $\mathrm{P}_{4} \times \mathrm{P}_{5}$ exhibited significant and positive superiority over SC 130 under both and across nitrogen levels.
\end{abstract}

Keywords: Maize, nitrogen levels, diallel analysis, gene action

\section{INTRODUCTION}

Maize (Zea mays L.) is the third important cereal crop in the world after wheat and rice (Adu et al., 2018). It is essential for human and animal fed. Also, it is used as a raw material for industrial products (El-Hosary et al., 2018). Therefore, increasing the productivity of such crop is the main goal of maize breeders in Egypt in order to decrease its import and respond to its high consumption (Kamara and Rehan, 2015). The knowledge of combining ability is important for selecting suitable parents for hybridization and identification of promising hybrids in breeding programs (Oyekunle et al., 2015). Diallel analysis is commonly used to gain information on gene action controlling traits of interest, and the combining ability of the parents (Griffing, 1956). Relative importance of both additive and non-additive gene effects is also estimated by diallel analysis. The additive gene effects have been reported to be important in the genetic expression of maize grain yield (Badu-Apraku and Oyekunle, 2012; Abd ElMottalb et al., 2013; Abo El-Haress, 2015; El-Hosary et al., 2018). However, other researchers reported that the non-additive genetic effects were represented the major role in the inheritance of maize grain yield and most of its components (Makumbi et al., 2011; Attia et al., 2015; Kamara, 2015; Wani et al, 2017). There is no agreement among the researchers on the type of gene action controlling maize yield or its related characters, and this due to differences in the genetic materials and the environments under which the experiments were performed. Much effort has been dedicated to estimate the interactions between genetic components and environments. Nitrogen $(\mathrm{N})$ is a vital nutrient required for the maize plants growth, metabolically active and photosynthesis (Haque et al., 2001). Abd El -Aty and Darwish (2006), Mosa et al. (2010) and Kamara et al. (2014) obtained significant GCA $\times \mathrm{N}$ and SCA $\times \mathrm{N}$ interactions for grain yield and other traits, an indication of variation of general and specific coming ability under different environments. This study was designed to estimate general (GCA) and specific (SCA) combining ability effects and their interactions with nitrogen levels as well as identify type of gene action controlling the inheritance of the studied traits.

\section{MATERIALS AND METHODS}

\section{Plant materials:}

Seven white inbred lines of maize (Zea mays L.) were used as parents in this study. The parental codes, names and sources of these inbred lines are presented in Table (1).

In 2016 growing season, all possible combinations excluding reciprocals were made among the seven inbred lines giving a total of $21 \mathrm{~F} 1$ crosses. In 2017 growing season, the resulted $21 \mathrm{~F} 1$ hybrids and the commercial check hybrid SC130 were evaluated in two separate experiments represented two different nitrogen levels; 90 (N1) and 120 (N2) kg N/fed at the Experimental Farm, Faculty of Agriculture, Kafrelsheikh University, Egypt. A randomized complete block design with three replications was used for each experiment.

\footnotetext{
"Corresponding author e-mail: Saok2006@yahoo.com
} 
Each plot consisted of two ridges, $5 \mathrm{~m}$ long and $0.70 \mathrm{~m}$ width. Planting was made in hills spaced at $0.25 \mathrm{~m}$ with three kernels per hill on one side of the ridge. The seedlings were thinned to one plant per hill after 21 days from planting. Nitrogen fertilizer was added in two equal doses before 1 st and $2^{\text {nd }}$ irrigations.

All other agricultural practices were carried out according to standard commercial recommendations for maize production.

Table (1): The code, name and pedigree of the parental maize inbred lines

\begin{tabular}{lcc}
\hline $\begin{array}{l}\text { Parent } \\
\text { code }\end{array}$ & Name & Source \\
\hline $\mathbf{P}_{\mathbf{1}}$ & Inb. 17 & Agricultural Research Center, Egypt \\
$\mathbf{P}_{\mathbf{2}}$ & Inb. 51 & Agricultural Research Center, Egypt \\
$\mathbf{P}_{\mathbf{3}}$ & Inb. 60 & Agricultural Research Center, Egypt \\
$\mathbf{P}_{\mathbf{4}}$ & Inb. 84 & Agricultural Research Center, Egypt \\
$\mathbf{P}_{\mathbf{5}}$ & Inb. 92 & Agricultural Research Center, Egypt \\
$\mathbf{P}_{\mathbf{6}}$ & Inb. 144 & Agricultural Research Center, Egypt \\
$\mathbf{P}_{\mathbf{7}}$ & CML23 & CIMMYT, Mexico \\
\hline
\end{tabular}

Data were collected for number of days from planting to $50 \%$ silking (day), plant height $(\mathrm{cm})$, ear height $(\mathrm{cm})$, ear length $(\mathrm{cm})$, ear diameter $(\mathrm{cm})$, number of rows/ear, number of kernels/row and grain yield ardab/feddan adjusted to $15.5 \%$ moisture content (one $\operatorname{ardab}=140 \mathrm{Kg}$, one feddan $=4200 \mathrm{~m}^{2}$ ). The obtained data were statistically analyzed for the analysis of variance according to Steel and Torrie (1980). The combined analysis was done whenever homogeneity of variance was detected. General and specific combining ability were estimated according to Griffing (1956), method-4, model-1.

\section{RESULTS AND DISCUSSION}

\section{Analysis of variance:}

The analysis of variance showed highly significant mean squares due to nitrogen levels $(\mathrm{N})$ for all the studied traits (Table 2), indicating overall differences between the two nitrogen levels. Genotypes (G) and crosses (Cr.) mean squares were found to be highly significant for all the studied traits at each and across nitrogen levels, indicating a wide diversity among the genetic materials used in the present study. Mean squares due to genotypes $\times$ nitrogen levels $(G \times$ $\mathrm{N})$ and crosses $\times$ nitrogen levels $(\mathrm{Cr} . \times \mathrm{N})$ interactions were significant for all the studied traits, revealing that the tested genotypes behaved differently from nitrogen level to another. These results corroborate the findings of Abd El-Aty et al. (2014) and Al-Naggar et al. (2015). Mean squares due to crosses vs. check were significant for all the studied traits, except days to 50\% silking under N2 level and the combined data, plant height and ear diameter under both nitrogen levels and the combined data and No. of rows/ear under N2 level. Insignificant interaction mean squares between crosses vs. check and nitrogen levels were observed for all the studied traits, except plant height and ear length. This result suggests that the heterotic effects were not differed by nitrogen levels changes. Mosa et al. (2010), El-Badawy (2013) and Katta et al. (2013) reached to the same conclusion for grain yield and most of its components.

\section{Mean performance:}

Mean performance of the $21 \mathrm{~F}_{1}$ crosses and the check hybrid SC130 for all the studied traits under both and across nitrogen levels are shown in Table (3).

In general, the mean values of the $21 \mathrm{~F}_{1}$ crosses and the check SC130 were higher under the N2 level $(120 \mathrm{~kg} \mathrm{~N} / \mathrm{fed})$ as compared to those under N1 level (90 kg N /fed) for all the studied traits, except days to $50 \%$ silking. The increase in mean performance of these traits at high nitrogen level might be attributed to the simulating effect of nitrogen on metabolic process in maize plants. According to Kaur et al. (2012) the increase in maize plant height with increase nitrogen level was due to the positive effect of nitrogen element on plant growth that leads to progressive increase in internodes length and consequently plant height. The increase in maize grain yield is largely due to an increase in metabolic process which in turn stimulates growth and increase total dry matter distributed to the grain and ultimately increase the most of yield components (Medici et al., 2004; Ngaboyisonga et al., 2009; Adu et al., 2018). Concerning the performance of the $\mathrm{F}_{1}$ crosses in comparison with the check hybrid SC130, data in Table (3) showed that, the crosses $\mathrm{P}_{1} \times \mathrm{P}_{3}, \quad \mathrm{P}_{1} \times \mathrm{P}_{4}$ and $\mathrm{P}_{1} \times \mathrm{P}_{7}$ under $\mathrm{N} 2$ level and the combined data and $\mathrm{P}_{1} \times \mathrm{P}_{5}, \mathrm{P}_{1} \times \mathrm{P}_{6}$ and $\mathrm{P}_{2} \times \mathrm{P}_{7}$ under both and across nitrogen levels were found to be significantly earlier than the check hybrid SC130. Earliness in maize is favorable character for saving water irrigation and escaping destructive injuries caused by the stem corn borers. Three crosses $\mathrm{P} 1 \times \mathrm{P} 6$, $\mathrm{P}_{2} \times \mathrm{P}_{6}$ and $\mathrm{P}_{5} \times \mathrm{P}_{6}$ at both nitrogen levels and the combined data were exhibited significantly deceased values as compared to the check hybrid $\mathrm{SC} 130$ for plant height. Also, the crosses i.e., $\mathrm{P}_{2} \times \mathrm{P}_{4}$ and $\mathrm{P}_{3} \times \mathrm{P}_{4}$ at $\mathrm{N} 1$ and $\mathrm{P}_{1} \times \mathrm{P}_{2}, \mathrm{P}_{2} \times \mathrm{P}_{5}, \mathrm{P}_{2} \times \mathrm{P}_{7}, \mathrm{P}_{5} \times \mathrm{P}_{7}$ and $\mathrm{P}_{6} \times \mathrm{P}_{7}$ at $\mathrm{N} 2$ and combined data were significantly shorter than the check hybrid SC130. With respect to ear height, the ten crosses $\mathrm{P}_{1} \times \mathrm{P}_{6}, \quad \mathrm{P}_{2} \times \mathrm{P}_{4}, \quad \mathrm{P}_{2} \times \mathrm{P}_{5}, \quad \mathrm{P}_{2} \times \mathrm{P}_{6} \quad \mathrm{P}_{2} \times \mathrm{P}_{7}, \quad \mathrm{P}_{3} \times \mathrm{P}_{4}$, $\mathrm{P}_{4} \times \mathrm{P}_{6}, \mathrm{P}_{4} \times \mathrm{P}_{7}, \mathrm{P}_{5} \times \mathrm{P}_{6}$ and $\mathrm{P}_{5} \times \mathrm{P}_{7}$ at both and across nitrogen levels had significantly lower ear placement than the check hybrid SC130. Concerning ear length, the three crosses $\mathrm{P}_{1} \times \mathrm{P}_{5}, \mathrm{P}_{2} \times \mathrm{P}_{7}$ and $\mathrm{P}_{4} \times \mathrm{P}_{5}$ under both nitrogen levels and the combined data significantly surpassed the check hybrid SC130. Regarding ear diameter, the cross $\mathrm{P}_{6} \times \mathrm{P}_{7}$ at the combined data and the four crosses $\mathrm{P}_{1} \times \mathrm{P}_{4}, \mathrm{P}_{1} \times \mathrm{P}_{5}, \mathrm{P}_{2} \times \mathrm{P}_{7}$ and $\mathrm{P}_{4} \times \mathrm{P}_{5}$ at both and across nitrogen levels were significantly superior than the check SC130. The three crosses $\mathrm{P}_{3} \times \mathrm{P}_{6}, \mathrm{P}_{4} \times \mathrm{P}_{5}$ and $\mathrm{P}_{4} \times \mathrm{P}_{6}$ gave the highest mean value for No. of rows/ear and significantly surpassed the check hybrid SC130. 
Table (2): Mean squares from ordinary and combining ability analysis for all the studied traits under the two nitrogen levels as well as the combined analysis across them

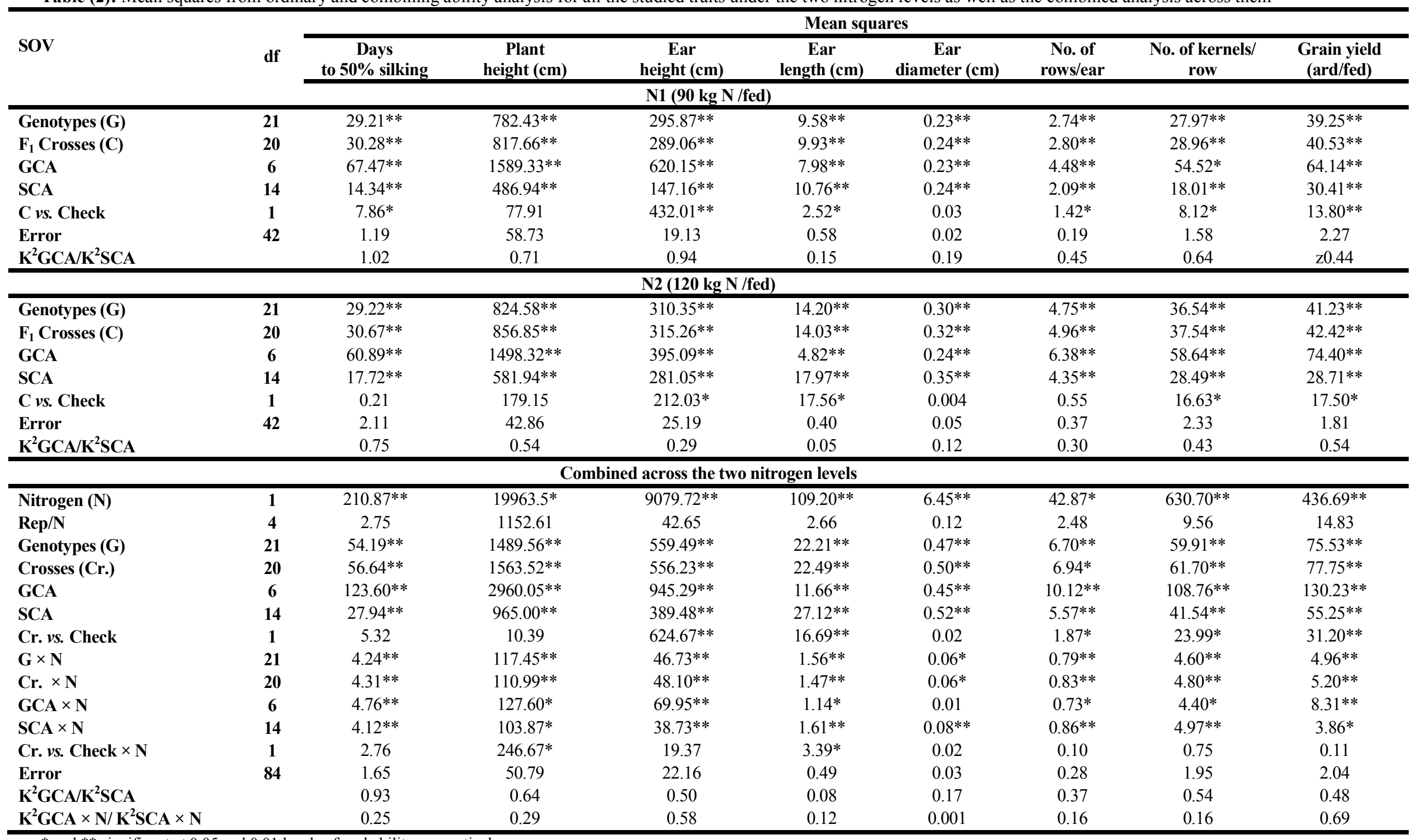

$*$ and ${ }^{* *}$ significant at 0.05 and 0.01 levels of probability, respectively. 
The two crosses $\mathrm{P}_{2} \times \mathrm{P}_{7}$ and $\mathrm{P}_{4} \times \mathrm{P}_{5}$ under nitrogen levels and the combined data as well as the cross $\mathrm{P}_{2} \times \mathrm{P}_{4}$ under N1 significantly possessed higher No. of kernels/row than the check hybrid SC130. Superiority percentage for grain yield (ard/fed) relative to the check hybrid SC130 (Table 3) revealed that the cross $\mathrm{P}_{3} \times \mathrm{P}_{6}$ under $\mathrm{N} 1$ and the two crosses $\mathrm{P}_{2} \times \mathrm{P}_{7}$ and $\mathrm{P}_{4} \times \mathrm{P}_{5}$ under both nitrogen levels and the combined data had positive and significant superiority percentage over the check hybrid SC130. The crosses $\mathrm{P}_{1} \times \mathrm{P}_{3}, \mathrm{P}_{2} \times \mathrm{P}_{3}$ and $\mathrm{P}_{2} \times \mathrm{P}_{6}$ gave positive superiority percentage over the check hybrid SC130, but it was not significant. Therefore, it could be concluded that these crosses could be efficient and prospective in maize breeding programs since they showed high values for grain yield and one or more of yield components traits. These results are in harmony with those obtained by ElBadawy (2013), El-Shamarka et al. (2015), El-Hosary et al. (2018). They found positive and significant superiority percentages compared to the check hybrids for maize grain yield. The fluctuation of hybrids performance from nitrogen level to another was detected for most traits. These results could be due to significant interaction between crosses and nitrogen levels.

Table (3): Mean performance of the $21 \mathrm{~F}_{1}$ crosses for all the studied traits under the two nitrogen levels and their combined data as well as superiority percentage relative to check hybrid SC130 for grain yield trait

\begin{tabular}{|c|c|c|c|c|c|c|c|c|c|c|c|c|}
\hline \multirow{2}{*}{ Crosses } & \multicolumn{3}{|c|}{ Days to $50 \%$ silking } & \multicolumn{3}{|c|}{ Plant height (cm) } & \multicolumn{3}{|c|}{ Ear height $(\mathrm{cm})$} & \multicolumn{3}{|c|}{ Ear length (cm) } \\
\hline & N1 & N2 & Comb. & N1 & $\mathbf{N 2}$ & Comb. & N1 & N2 & Comb. & N1 & $\mathbf{N 2}$ & Comb. \\
\hline $\mathbf{P}_{1} \times \mathbf{P}_{2}$ & 62.0 & 60.7 & 61.3 & 198.8 & 230.7 & 214.8 & 104.6 & 119.8 & 112.2 & 18.5 & 20.1 & 19.3 \\
\hline $\mathbf{P}_{1} \times \mathbf{P}_{3}$ & 61.5 & 58.8 & 60.2 & 243.8 & 267.5 & 255.7 & 131.5 & 144.0 & 137.8 & 15.0 & 17.6 & 16.3 \\
\hline $\mathbf{P}_{1} \times \mathbf{P}_{4}$ & 63.5 & 58.3 & 60.9 & 241.3 & 255.7 & 248.5 & 124.0 & 146.0 & 135.0 & 16.0 & 18.0 & 17.0 \\
\hline $\mathbf{P}_{1} \times \mathbf{P}_{5}$ & 61.0 & 57.0 & 59.0 & 248.9 & 270.3 & 259.6 & 124.3 & 137.3 & 130.8 & 19.8 & 22.6 & 21.2 \\
\hline $\mathbf{P}_{1} \times \mathbf{P}_{6}$ & 59.0 & 58.0 & 58.5 & 190.7 & 210.9 & 200.8 & 102.1 & 111.0 & 106.6 & 16.5 & 17.0 & 16.8 \\
\hline $\mathbf{P}_{1} \times \mathbf{P}_{7}$ & 61.5 & 58.5 & 60.0 & 233.5 & 263.8 & 248.7 & 134.8 & 148.0 & 141.4 & 18.8 & 19.0 & 18.9 \\
\hline $\mathbf{P}_{2} \times \mathbf{P}_{3}$ & 68.9 & 66.7 & 67.8 & 213.8 & 265.3 & 239.6 & 115.5 & 143.5 & 129.5 & 18.8 & 20.0 & 19.4 \\
\hline $\mathbf{P}_{2} \times \mathbf{P}_{4}$ & 64.7 & 61.5 & 63.1 & 215.6 & 231.3 & 223.5 & 113.0 & 132.3 & 122.7 & 16.0 & 17.2 & 16.6 \\
\hline $\mathbf{P}_{2} \times \mathbf{P}_{5}$ & 63.5 & 60.9 & 62.2 & 208.8 & 224.8 & 216.8 & 116.8 & 128.5 & 122.6 & 17.0 & 18.0 & 17.5 \\
\hline $\mathbf{P}_{2} \times \mathbf{P}_{6}$ & 65.2 & 64.3 & 64.8 & 190.2 & 223.8 & 207.0 & 100.5 & 127.3 & 113.9 & 19.0 & 20.0 & 19.5 \\
\hline $\mathbf{P}_{2} \times \mathbf{P}_{7}$ & 60.5 & 59.3 & 59.9 & 202.9 & 225.6 & 214.3 & 110.5 & 124.0 & 117.3 & 19.7 & 22.6 & 21.2 \\
\hline $\mathbf{P}_{3} \times \mathbf{P}_{4}$ & 66.3 & 60.5 & 63.4 & 218.8 & 235.5 & 227.2 & 118.5 & 131.3 & 124.9 & 15.0 & 16.0 & 15.5 \\
\hline $\mathbf{P}_{3} \times \mathbf{P}_{5}$ & 67.4 & 64.8 & 66.1 & 217.5 & 241.2 & 229.4 & 129.3 & 144.0 & 136.6 & 14.0 & 15.6 & 14.8 \\
\hline $\mathbf{P}_{3} \times \mathbf{P}_{6}$ & 66.0 & 66.3 & 66.1 & 230.0 & 250.0 & 240.0 & 123.0 & 147.3 & 135.1 & 18.0 & 21.2 & 19.6 \\
\hline $\mathbf{P}_{3} \times \mathbf{P}_{7}$ & 63.5 & 61.7 & 62.6 & 217.8 & 256.3 & 237.1 & 120.5 & 142.3 & 131.4 & 17.5 & 20.5 & 19.0 \\
\hline $\mathbf{P}_{4} \times \mathbf{P}_{5}$ & 68.5 & 66.0 & 67.3 & 221.3 & 243.8 & 232.6 & 128.0 & 140.0 & 134.0 & 19.8 & 23.1 & 21.5 \\
\hline $\mathbf{P}_{4} \times \mathbf{P}_{6}$ & 68.8 & 67.3 & 68.1 & 215.0 & 238.8 & 226.9 & 110.5 & 128.0 & 119.3 & 16.6 & 18.0 & 17.3 \\
\hline $\mathbf{P}_{4} \times \mathbf{P}_{7}$ & 65.0 & 63.0 & 64.0 & 212.5 & 238.3 & 225.4 & 108.0 & 121.0 & 114.5 & 18.3 & 20.0 & 19.2 \\
\hline $\mathbf{P}_{5} \times \mathbf{P}_{6}$ & 70.3 & 63.5 & 66.9 & 196.7 & 222.5 & 209.6 & 106.8 & 126.9 & 116.8 & 14.7 & 18.1 & 16.4 \\
\hline $\mathbf{P}_{5} \times \mathbf{P}_{7}$ & 67.9 & 65.5 & 66.7 & 206.8 & 235.0 & 220.9 & 121.0 & 132.8 & 126.9 & 15.6 & 18.2 & 16.9 \\
\hline $\mathbf{P}_{6} \times \mathbf{P}_{7}$ & 62.8 & 60.9 & 61.9 & 205.3 & 227.5 & 216.4 & 112.0 & 136.5 & 124.3 & 15.8 & 16.7 & 16.3 \\
\hline Crosses mean & 64.7 & 62.1 & 63.4 & 215.7 & 240.9 & 228.3 & 116.9 & 133.9 & 125.4 & 17.2 & 19.0 & 18.1 \\
\hline Check SC130 & 63.0 & 61.8 & 62.4 & 210.5 & 248.8 & 229.7 & 129.2 & 142.5 & 135.9 & 18.1 & 21.5 & 19.8 \\
\hline LSD 0.05 & 1.8 & 2.4 & 1.5 & 12.6 & 10.8 & 8.2 & 7.2 & 8.3 & 5.4 & 1.3 & 1.0 & 0.8 \\
\hline LSD 0.01 & 2.4 & 3.2 & 2.0 & 16.9 & 14.5 & 10.9 & 9.7 & 11.1 & 7.2 & 1.7 & 1.4 & 1.1 \\
\hline
\end{tabular}


Table (3): Cont.

\begin{tabular}{|c|c|c|c|c|c|c|c|c|c|c|c|c|c|c|c|}
\hline \multirow{2}{*}{ Crosses } & \multicolumn{3}{|c|}{$\begin{array}{l}\text { Ear diameter } \\
(\mathrm{cm})\end{array}$} & \multicolumn{3}{|c|}{ No. of rows/ear } & \multicolumn{3}{|c|}{$\begin{array}{c}\text { No. of } \\
\text { kernels/row }\end{array}$} & \multicolumn{3}{|c|}{$\begin{array}{l}\text { Grain yield } \\
\text { (ard/fed) }\end{array}$} & \multicolumn{3}{|c|}{$\begin{array}{c}\text { Superiority \% } \\
\text { relative to SC130 }\end{array}$} \\
\hline & N1 & $\mathbf{N} 2$ & Comb. & N1 & $\mathbf{N 2}$ & Comb & N1 & $\mathbf{N 2}$ & Comb & N1 & $\mathbf{N 2}$ & Comb & N1 & $\mathbf{N 2}$ & Comb \\
\hline $\mathbf{P}_{1} \times \mathbf{P}_{2}$ & 4.6 & 5.2 & 4.9 & 12.3 & 13.6 & 13.0 & 34.3 & 38.0 & 36.2 & 24.0 & 31.6 & 27.8 & $-12.7^{* *}$ & 0.3 & $-5.8^{*}$ \\
\hline $\mathbf{P}_{1} \times \mathbf{P}_{3}$ & 4.8 & 5.0 & 4.9 & 13.3 & 14.2 & 13.8 & 30.0 & 33.0 & 31.5 & 27.6 & 31.5 & 29.5 & 0.3 & 0.0 & 0.2 \\
\hline $\mathbf{P}_{1} \times \mathbf{P}_{4}$ & 5.0 & 5.5 & 5.3 & 13.0 & 14.2 & 13.6 & 35.0 & 41.0 & 38.0 & 21.0 & 28.6 & 24.8 & $-23.6^{* *}$ & $-9.1^{*}$ & $-15.9^{* *}$ \\
\hline $\mathbf{P}_{1} \times \mathbf{P}_{5}$ & 5.0 & 5.5 & 5.3 & 13.7 & 14.0 & 13.9 & 31.5 & 38.0 & 34.8 & 19.5 & 22.4 & 21.0 & $-29.1^{* *}$ & $-28.9^{* *}$ & $-29.0^{* *}$ \\
\hline $\mathbf{P}_{1} \times \mathbf{P}_{6}$ & 4.2 & 4.7 & 4.5 & 13.5 & 14.0 & 13.8 & 32.0 & 39.5 & 35.8 & 19.5 & 21.0 & 20.3 & $-29.0^{* *}$ & $-33.3^{* *}$ & $-31.3^{* *}$ \\
\hline $\mathbf{P}_{1} \times \mathbf{P}_{7}$ & 4.8 & 5.1 & 5.0 & 11.7 & 13.0 & 12.4 & 28.0 & 30.0 & 29.0 & 21.0 & 24.0 & 22.5 & $-23.6^{* *}$ & $-23.8^{* *}$ & $-23.7^{* *}$ \\
\hline $\mathbf{P}_{2} \times \mathbf{P}_{3}$ & 4.2 & 4.9 & 4.6 & 12.3 & 15.0 & 13.7 & 36.8 & 38.0 & 37.4 & 28.4 & 33.5 & 31.0 & 3.3 & 6.3 & 4.9 \\
\hline $\mathbf{P}_{2} \times \mathbf{P}_{4}$ & 4.6 & 4.9 & 4.8 & 14.0 & 14.5 & 14.3 & 37.2 & 40.0 & 38.6 & 27.6 & 30.0 & 28.8 & 0.4 & -4.8 & -2.4 \\
\hline $\mathbf{P}_{2} \times \mathbf{P}_{5}$ & 4.5 & 4.6 & 4.6 & 13.0 & 14.0 & 13.5 & 33.2 & 41.0 & 37.1 & 23.3 & 30.7 & 27.0 & $-15.3^{* *}$ & -2.5 & $-8.5^{* *}$ \\
\hline $\mathbf{P}_{2} \times \mathbf{P}_{6}$ & 4.3 & 4.7 & 4.5 & 13.3 & 14.3 & 13.8 & 33.2 & 39.5 & 36.4 & 27.5 & 32.0 & 29.8 & 0.0 & 1.6 & 0.8 \\
\hline $\mathbf{P}_{2} \times \mathbf{P}_{7}$ & 5.0 & 5.5 & 5.3 & 14.0 & 15.9 & 15.0 & 39.0 & 43.0 & 41.0 & 30.6 & 34.5 & 32.6 & $11.3^{*}$ & $9.5^{* *}$ & $10.3^{* *}$ \\
\hline $\mathbf{P}_{3} \times \mathbf{P}_{4}$ & 4.6 & 4.9 & 4.8 & 14.7 & 15.6 & 15.2 & 35.8 & 39.7 & 37.8 & 25.0 & 29.0 & 27.0 & $-9.1^{*}$ & $-7.9^{*}$ & -8.5 \\
\hline $\mathbf{P}_{3} \times \mathbf{P}_{5}$ & 4.7 & 5.1 & 4.9 & 13.0 & 13.0 & 13.0 & 34.3 & 37.8 & 36.1 & 20.0 & 24.0 & 22.0 & $-27.3^{* *}$ & $-23.8^{* *}$ & $-25.4^{* *}$ \\
\hline $\mathbf{P}_{3} \times \mathbf{P}_{6}$ & 4.8 & 5.2 & 5.0 & 15.0 & 18.0 & 16.5 & 34.7 & 39.0 & 36.9 & 30.6 & 31.5 & 31.1 & $11.4^{*}$ & -0.1 & 5.3 \\
\hline $\mathbf{P}_{3} \times \mathbf{P}_{7}$ & 4.6 & 5.1 & 4.9 & 12.7 & 13.0 & 12.9 & 29.5 & 35.0 & 32.3 & 26.0 & 28.8 & 27.4 & -5.5 & $-8.6^{*}$ & $-7.1^{*}$ \\
\hline $\mathbf{P}_{4} \times \mathbf{P}_{5}$ & 5.0 & 5.9 & 5.5 & 14.9 & 16.1 & 15.5 & 39.0 & 44.5 & 41.8 & 30.7 & 34.0 & 32.4 & $11.6^{*}$ & $7.9^{*}$ & $9.7^{* *}$ \\
\hline $\mathbf{P}_{4} \times \mathbf{P}_{6}$ & 4.1 & 4.7 & 4.4 & 14.9 & 16.2 & 15.6 & 32.0 & 37.8 & 34.9 & 27.3 & 29.9 & 28.6 & -0.7 & -5.1 & -3.1 \\
\hline $\mathbf{P}_{4} \times \mathbf{P}_{7}$ & 4.8 & 4.9 & 4.9 & 14.0 & 15.0 & 14.5 & 32.0 & 35.0 & 33.5 & 28.6 & 31.0 & 29.8 & 4.0 & -1.6 & 1.0 \\
\hline $\mathbf{P}_{5} \times \mathbf{P}_{6}$ & 4.6 & 5.1 & 4.9 & 12.0 & 13.0 & 12.5 & 31.8 & 35.0 & 33.4 & 24.4 & 27.1 & 25.8 & $-11.2^{*}$ & $-14.0^{* *}$ & $-12.7^{* *}$ \\
\hline $\mathbf{P}_{5} \times \mathbf{P}_{7}$ & 4.4 & 5.1 & 4.8 & 12.7 & 13.9 & 13.3 & 28.8 & 32.0 & 30.4 & 25.7 & 28.5 & 27.1 & -6.5 & $-9.5^{* *}$ & $-8.1^{* *}$ \\
\hline $\mathbf{P}_{6} \times \mathbf{P}_{7}$ & 4.9 & 5.4 & 5.2 & 13.3 & 15.3 & 14.3 & 31.5 & 36.8 & 34.2 & 23.0 & 26.0 & 24.5 & $-16.4^{* *}$ & $-17.5^{* *}$ & $-16.9^{* *}$ \\
\hline Crosses mean & 4.6 & 5.1 & 4.9 & 13.4 & 14.6 & 14.0 & 33.3 & 37.8 & 35.6 & 25.3 & 29.0 & 27.2 & - & - & - \\
\hline Check SC130 & 4.8 & 5.1 & 4.9 & 14.1 & 15.0 & 14.6 & 35.0 & 40.2 & 37.6 & 27.5 & 31.5 & 29.5 & - & - & - \\
\hline LSD 0.05 & 0.2 & 0.4 & 0.2 & 0.7 & 1.0 & 0.6 & 2.1 & 2.5 & 1.6 & 2.5 & 2.2 & 1.6 & - & - & - \\
\hline LSD 0.01 & 0.3 & 0.5 & 0.3 & 1.0 & 1.3 & 0.8 & 2.8 & 3.4 & 2.1 & 3.3 & 3.0 & 2.2 & - & - & - \\
\hline
\end{tabular}

\section{Combining ability:}

Mean squares due to general combining ability (GCA) and specific combining ability (SCA) were highly significant for all the studied traits under both nitrogen levels and the combined analysis (Table 2), revealing that both additive and non-additive gene effects were important in the inheritance of these traits. The GCA/SCA ratio was less than unity for all the studied traits under both nitrogen levels and their combined data, except days to $50 \%$ silking under $\mathrm{N} 1$ level $(90 \mathrm{~kg} \mathrm{~N} / \mathrm{fed})$, indicating that these traits were predominantly controlled by the non-additive gene action. These results are in accordance with those obtained by San et al. (2001), Mosa (2003), Ahmed (2013) and El-Ghonemy (2015) for plant height, Mosa (2010), Katta et al. (2013) and El-Hosary (2014) for ear diameter and Attia et al. (2015), Hassan (2015) and Wani et al. (2017) for grain yield. For the exceptional trait, the ratio of GCA/GCA was equal unity, indicating that additive and non-additive type of gene action have the same importance in the performance of this trait. Significant interaction mean square between nitrogen levels and both types of combining ability $(\mathrm{GCA} \times \mathrm{N}$ and $\mathrm{SCA} \times \mathrm{N})$ were detected for all the studied traits, except GCA $\times \mathrm{N}$ for ear diameter. These results suggested that the behavior of the two types of gene action (additive and nonadditive) varied from nitrogen level to another. It is fairly evident that the ratio of GCA $\times \mathrm{N} / \mathrm{SCA} \times \mathrm{N}$ was less than unity for all the studied traits. This result indicated that the non-additive effects were more affected by nitrogen levels than the additive genetic effects. In this respect Mosa et al. (2010) and Kamara (2015) reported that the non-additive genetic effects were more influenced by nitrogen levels than additive gene actions for grain yield and most of its components. 
General combining ability (GCA) effects:

Estimates of general combining ability $\left(\hat{g}_{i}\right)$ effects of the seven inbred lines under both and across nitrogen levels are shown in Table (4). High positive values of $\left(\hat{g}_{i}\right)$ effects would be of interest for all studied traits, except days to $50 \%$ silking, plant and ear heights where high negative values would be favored. The parental inbred line $\mathrm{P}_{1}$ showed significant negative $\left(\hat{g}_{i}\right)$ effects for days to $50 \%$ silking and showed significant positive effects for ear diameter under both nitrogen levels and the combined data. The parental inbred line $\mathrm{P}_{2}$ showed significant negative $\left(\hat{g}_{i}\right)$ effects for plant and ear heights and gave significant positive effects for ear length, No. of kernels/row and grain yield under both under both nitrogen levels and the combined data. The parental inbred line $\mathrm{P}_{3}$ expressed significant positive $\left(\hat{g}_{i}\right)$ effects for grain yield under both and across nitrogen levels. However, it gave significant undesirable or insignificant $\left(\hat{g}_{i}\right)$ effects for other traits. The parental inbred line $\mathrm{P}_{4}$ seemed to be suitable combiner for No. of rows/ear, No. of kernels/row and grain yield under both and across nitrogen levels, due to its positive and significant $\left(\hat{g}_{i}\right)$ values in this concern. The parental inbred line $\mathrm{P}_{5}$ behaved as a good combiner for ear diameter. The parental inbred line $\mathrm{P}_{6}$ gave highly significant negative $\left(\hat{g}_{i}\right)$ effects for plant and ear heights as well as showed significant positive $\left(\hat{g}_{i}\right)$ effects for ear length and No. of rows/ear under both and across nitrogen levels. The parental inbred line $\mathrm{P}_{7}$ expressed significant negative $\left(\hat{g}_{i}\right)$ effects for days to $50 \%$ silking and exhibited significant positive $\left(\hat{g}_{i}\right)$ effects for ear length and ear diameter under both nitrogen levels and the combined data.

Table (4): General combining ability $\left(\hat{g}_{i}\right)$ effects of the seven inbred lines for all the studied traits under each and across nitrogen levels

\begin{tabular}{|c|c|c|c|c|c|c|c|c|c|c|c|c|}
\hline \multirow{2}{*}{ Inbred line } & \multicolumn{3}{|c|}{ Ear diameter (cm) } & \multicolumn{3}{|c|}{ No. of rows/ear } & \multicolumn{3}{|c|}{ No. of kernels/row } & \multicolumn{3}{|c|}{ Grain yield (ard/fed) } \\
\hline & N1 & N2 & Comb. & N1 & N2 & Comb. & N1 & N2 & Comb. & N1 & N2 & Comb. \\
\hline $\mathbf{P}_{1}$ & $-3.89 * *$ & $-4.23 * *$ & $-4.06^{* *}$ & $12.55^{* *}$ & $10.73 * *$ & $11.64^{* *}$ & $3.96^{* *}$ & 0.55 & $2.25^{*}$ & 0.33 & 0.03 & 0.18 \\
\hline $\mathbf{P}_{2}$ & -0.52 & 0.19 & -0.18 & $-12.83^{* *}$ & $-8.75^{* *}$ & $-10.79 * *$ & $-8.11 * *$ & $-5.59 * *$ & $-6.85^{* *}$ & $1.21 * *$ & $0.75^{* *}$ & $0.98 * *$ \\
\hline $\mathbf{P}_{3}$ & $1.13^{* *}$ & $1.28 * *$ & $1.20^{* *}$ & $9.48^{* *}$ & $14.09 * *$ & $11.79 * *$ & $7.37 * *$ & $9.79 * *$ & $8.58^{* *}$ & $-0.93 * *$ & $-0.65 * *$ & $-0.79 * *$ \\
\hline $\mathbf{P}_{4}$ & $1.77 * *$ & $0.84 *$ & $1.30 * *$ & $6.04 * *$ & -0.39 & 2.83 & 0.12 & -0.95 & -0.42 & -0.25 & $-0.37^{*}$ & -0.31 \\
\hline $\mathbf{P}_{5}$ & $2.13^{* *}$ & $1.06^{* *}$ & $1.59 * *$ & 1.14 & -1.55 & -0.20 & $4.92 * *$ & 1.23 & $3.07 * *$ & $-0.41 *$ & 0.29 & -0.06 \\
\hline $\mathbf{P}_{6}$ & $0.83 * *$ & $1.58^{* *}$ & $1.20^{* *}$ & $-13.28 * *$ & $-14.37 * *$ & $-13.82 * *$ & $-9.31 * *$ & $-5.28 * *$ & $-7.30^{* *}$ & $-0.47 *$ & $-0.63 * *$ & $-0.55 * *$ \\
\hline $\mathbf{P}_{7}$ & $-1.45^{* *}$ & $-0.70^{*}$ & $-1.08 * *$ & -3.10 & 0.23 & -1.43 & 1.07 & 0.25 & 0.66 & $0.55^{* *}$ & $0.57 * *$ & $0.56^{* *}$ \\
\hline $\operatorname{LSD}(0.05)_{\mathrm{gi}}$ & 0.53 & 0.70 & 0.61 & 3.70 & 3.16 & 3.39 & 2.11 & 2.42 & 2.24 & 0.37 & 0.31 & 0.33 \\
\hline $\operatorname{LSD}(0.01)_{g i}$ & 0.71 & 0.94 & 0.81 & 4.95 & 4.23 & 4.49 & 2.83 & 3.24 & 2.97 & 0.49 & 0.41 & 0.44 \\
\hline LSD (0.05) gi-gi & 0.81 & 1.07 & 0.93 & 5.66 & 4.83 & 5.18 & 3.23 & 3.70 & 3.42 & 0.56 & 0.47 & 0.51 \\
\hline LSD (0.01) gi-gj & 1.08 & 1.43 & 1.24 & 7.57 & 6.46 & 6.87 & 4.32 & 4.96 & 4.53 & 0.75 & 0.62 & 0.68 \\
\hline \multicolumn{13}{|l|}{ Table (4): Cont. } \\
\hline \multirow{2}{*}{ Inbred line } & \multicolumn{3}{|c|}{ Ear diameter (cm) } & \multicolumn{3}{|c|}{ No. of rows/ear } & \multicolumn{3}{|c|}{ No. of kernels/row } & \multicolumn{3}{|c|}{ Grain yield (ard/fed) } \\
\hline & N1 & $\mathbf{N 2}$ & Comb. & N1 & $\mathbf{N 2}$ & Comb. & N1 & $\mathbf{N 2}$ & Comb. & N1 & $\mathbf{N 2}$ & Comb. \\
\hline $\mathbf{P}_{1}$ & $0.11^{* *}$ & $0.11^{*}$ & $0.105^{* *}$ & $-0.57 * *$ & $-0.87 * *$ & $-0.72 * *$ & $-1.81^{* *}$ & $-1.45^{* *}$ & $-1.63^{* *}$ & $-3.84^{* *}$ & $-3.01 * *$ & $-3.43 * *$ \\
\hline $\mathbf{P}_{2}$ & $-0.13 * *$ & $-0.15 * *$ & $-0.14 * *$ & $-0.29 * *$ & -0.01 & -0.15 & $2.77 * *$ & $2.55^{* *}$ & $2.66^{* *}$ & $1.91 * *$ & $3.62 * *$ & $2.77 * *$ \\
\hline $\mathbf{P}_{3}$ & -0.03 & -0.07 & -0.05 & 0.13 & 0.29 & 0.21 & 0.24 & $-0.85^{*}$ & -0.30 & $1.16^{* *}$ & 0.82 & $0.99 * *$ \\
\hline $\mathbf{P}_{4}$ & 0.05 & 0.05 & 0.05 & $1.02 * *$ & $0.85^{* *}$ & $0.93 * *$ & $2.22 * *$ & $2.25^{* *}$ & $2.24 * *$ & $1.67 * *$ & $1.67 * *$ & $1.67^{* *}$ \\
\hline $\mathbf{P}_{5}$ & $0.07 *$ & $0.15^{* *}$ & $0.11^{* *}$ & $-0.22 *$ & $-0.67 * *$ & $-0.45^{* *}$ & -0.26 & 0.31 & 0.03 & $-1.64 * *$ & $-1.49 * *$ & $-1.56^{* *}$ \\
\hline $\mathbf{P}_{6}$ & -0.19 & $-0.18 * *$ & $-0.19 * *$ & $0.33 * *$ & $0.69 * *$ & $0.51^{* *}$ & $-0.94 * *$ & 0.17 & -0.38 & 0.11 & $-1.34 * *$ & -0.61 \\
\hline $\mathbf{P}_{7}$ & $0.13^{* *}$ & $0.11^{*}$ & $0.12 * *$ & $-0.39 * *$ & -0.25 & $-0.32 *$ & $-2.22 * *$ & $-2.99 * *$ & $-2.60 * *$ & 0.62 & -0.27 & 0.17 \\
\hline $\operatorname{LSD}(0.05)_{\mathrm{gi}}$ & 0.06 & 0.11 & 0.09 & 0.21 & 0.30 & 0.25 & 0.61 & 0.74 & 0.66 & 0.73 & 0.65 & 0.68 \\
\hline $\operatorname{LSD}(0.01)_{\mathrm{gi}}$ & 0.08 & 0.14 & 0.11 & 0.28 & 0.39 & 0.33 & 0.81 & 0.99 & 0.88 & 0.97 & 0.87 & 0.90 \\
\hline LSD (0.05) gi-gi & 0.10 & 0.16 & 0.13 & 0.32 & 0.45 & 0.39 & 0.93 & 1.13 & 1.02 & 1.11 & 0.99 & 1.04 \\
\hline $\operatorname{LSD}(0.01)_{g i-g i}$ & 0.13 & 0.22 & 0.17 & 0.43 & 0.60 & 0.51 & 1.24 & 1.51 & 1.35 & 1.49 & 1.33 & 1.38 \\
\hline
\end{tabular}


The recent results, pointed out that, the inbred lines $\mathrm{P}_{1}$ and $\mathrm{P}_{7}$ considered as combiners for the improvement of earliness. The inbred lines $\mathrm{P}_{2}$ and $\mathrm{P}_{6}$ for developing were short and lower ear placement hybrids. The inbred lines $\mathrm{P}_{2}, \mathrm{P}_{3}$ and $\mathrm{P}_{4}$ behaved as the appropriate combiner for grain yield and some of its components. Such obtained results indicated that these inbred lines possess favorable genes and that improvement in respective traits may be attained if they are incorporated in maize hybridization program. It is worth noting that the parental inbred line which possessed high $\left(\hat{g}_{i}\right)$ effects for grain yield exhibited desirable $\left(\hat{g}_{i}\right)$ effects for one or more of the traits contributing to grain yield. El-Shamarka et al. (2015) and El-Hosary et al. (2018) reported that $\left(\hat{g}_{i}\right)$ effects were desirable and significant for earliness, grain yield and its components.

\section{Specific combining ability (SCA) effects:}

Estimates of specific combining ability $\left(\hat{S}_{i j}\right)$ effects of the $21 \mathrm{~F} 1$ crosses for all the studied traits under the two nitrogen levels and their combined data are shown in Table (5). The most desirable and significant $\left(\hat{S}_{i j}\right)$ effects under both nitrogen levels and the combined data were obtained by the crosses; $\mathrm{P}_{1} \times \mathrm{P}_{5}$,
$\mathrm{P}_{2} \times \mathrm{P}_{4}, \mathrm{P}_{2} \times \mathrm{P}_{5}, \mathrm{P}_{2} \times \mathrm{P}_{7}, \mathrm{P}_{3} \times \mathrm{P}_{4}$, and $\mathrm{P}_{6} \times \mathrm{P}_{7}$ for days to $50 \%$ silking (towards earliness), $\mathrm{P}_{1} \times \mathrm{P}_{2}, \mathrm{P}_{1} \times \mathrm{P}_{6}, \mathrm{P}_{3} \times \mathrm{P}_{4}$ and $\mathrm{P}_{3} \times \mathrm{P}_{5}$ for plant height (towards shorter plants), $\mathrm{P}_{1} \times \mathrm{P} 2$, $\mathrm{P}_{1} \times \mathrm{P}_{6}, \mathrm{P}_{3} \times \mathrm{P}_{4}$ and $\mathrm{P}_{4} \times \mathrm{P}_{7}$ for ear height (towards lower ear placement), $\mathrm{P}_{1} \times \mathrm{P}_{5}, \mathrm{P}_{2} \times \mathrm{P}_{3}, \mathrm{P}_{2} \times \mathrm{P}_{6}, \mathrm{P}_{2} \times \mathrm{P}_{7}, \mathrm{P}_{3} \times \mathrm{P}_{6}$, $\mathrm{P}_{3} \times \mathrm{P}_{7}, \mathrm{P}_{4} \times \mathrm{P}_{5}$ and $\mathrm{P}_{4} \times \mathrm{P}_{7}$ for ear length, $\mathrm{P}_{1} \times \mathrm{P}_{4}, \mathrm{P}_{1} \times \mathrm{P}_{5}$, $\mathrm{P}_{2} \times \mathrm{P}_{7}, \mathrm{P}_{3} \times \mathrm{P}_{6}, \mathrm{P}_{4} \times \mathrm{P}_{5}$ and $\mathrm{P}_{6} \times \mathrm{P}_{7}$ for ear diameter, $\mathrm{P}_{1} \times \mathrm{P}_{5}$, $\mathrm{P}_{2} \times \mathrm{P}_{7}, \mathrm{P}_{3} \times \mathrm{P}_{6}$ and $\mathrm{P}_{4} \times \mathrm{P}_{5}$ for No. of rows/ear, $\mathrm{P}_{1} \times \mathrm{P}_{4}$, $\mathrm{P}_{1} \times \mathrm{P}_{6}, \quad \mathrm{P}_{2} \times \mathrm{P}_{7}, \mathrm{P}_{3} \times \mathrm{P}_{6}, \quad \mathrm{P} 4 \times \mathrm{P}_{5}$ and $\mathrm{P}_{6} \times \mathrm{P}_{7}$ for No. of kernels/row and $\mathrm{P}_{1} \times \mathrm{P}_{3}, \mathrm{P}_{2} \times \mathrm{P}_{7}, \mathrm{P}_{3} \times \mathrm{P}_{6}, \mathrm{P}_{4} \times \mathrm{P}_{5}$ and $\mathrm{P}_{5} \times \mathrm{P}_{7}$ for grain yield. The previous crosses might be of prime importance in breeding programs for traditional breeding procedures (EL-Hosary, 2014). It is notable that the crosses that showed high SCA effects for grain yield also showed high SCA effects for one or more traits of yield components. For example, the crosses $\mathrm{P}_{2} \times \mathrm{P}_{7}, \mathrm{P}_{3} \times \mathrm{P}_{6}$ and $\mathrm{P}_{4} \times \mathrm{P}_{5}$ which showed high SCA effects for grain yield also showed high SCA effects for ear length and No. of rows/ear No. of kernels/row. In most traits, the values of SCA effects were mostly different from nitrogen level to another. These findings coincided with that discussed elsewhere in this study where significant SCA by nitrogen levels mean squares were detected (Table 2).

Table (5): Estimates of specific combining ability $\left(\hat{S}_{i j}\right)$ effects of the $21 \mathrm{~F}_{1}$ crosses for all the studied traits under the two nitrogen levels and the combined data

\begin{tabular}{|c|c|c|c|c|c|c|c|c|c|c|c|c|}
\hline \multirow{2}{*}{ Crosses } & \multicolumn{3}{|c|}{ Days to $50 \%$ silking } & \multicolumn{3}{|c|}{ Plant height (cm) } & \multicolumn{3}{|c|}{ Ear height (cm) } & \multicolumn{3}{|c|}{ Ear length $(\mathrm{cm})$} \\
\hline & N1 & $\mathbf{N 2}$ & Comb. & N1 & $\mathbf{N 2}$ & Comb. & N1 & $\mathbf{N 2}$ & Comb. & N1 & N2 & Comb. \\
\hline $\mathbf{P}_{1} \times \mathbf{P}_{2}$ & $1.86^{* *}$ & $2.64 * *$ & $2.25 * *$ & $-16.60 * *$ & $-12.07 * *$ & $-14.34 * *$ & $-8.14 * *$ & $-9.05^{* *}$ & $-8.59 * *$ & -0.19 & 0.29 & 0.05 \\
\hline $\mathbf{P}_{1} \times \mathbf{P}_{3}$ & -0.40 & -0.32 & -0.36 & 6.06 & 1.79 & 3.92 & 3.27 & -0.22 & 1.53 & $-1.55^{* *}$ & $-0.81 * *$ & $-1.18^{* *}$ \\
\hline $\mathbf{P}_{1} \times \mathbf{P}_{4}$ & 0.96 & -0.38 & 0.29 & 7.00 & 4.47 & 5.73 & 3.02 & $12.52 * *$ & $7.77 * *$ & $-1.23^{* *}$ & $-0.69 *$ & $-0.96^{* *}$ \\
\hline $\mathbf{P}_{1} \times \mathbf{P}_{5}$ & $-1.90 * *$ & $-1.90 * *$ & $-1.90 * *$ & $19.50^{* *}$ & $20.23 * *$ & $19.86^{* *}$ & -1.53 & 1.59 & 0.03 & $2.73 * *$ & $3.25 * *$ & $2.99 * *$ \\
\hline $\mathbf{P}_{1} \times \mathbf{P}_{6}$ & $-2.60 * *$ & $-1.42 *$ & $-2.01 * *$ & $-24.28 * *$ & $-26.35^{* *}$ & $-25.32 * *$ & $-9.45^{* *}$ & $-18.15^{* *}$ & $-13.80 * *$ & -0.51 & $-1.43^{* *}$ & $-0.97 * *$ \\
\hline $\mathbf{P}_{1} \times \mathbf{P}_{7}$ & $2.08 * *$ & 1.36 & $1.72 * *$ & $8.34^{*}$ & $11.95^{* *}$ & $10.14^{* *}$ & $12.82 * *$ & $13.32 * *$ & $13.07 * *$ & $0.77 *$ & $-0.63 *$ & 0.07 \\
\hline $\mathbf{P}_{2} \times \mathbf{P}_{3}$ & $3.74 * *$ & $3.16^{* *}$ & $3.45^{* *}$ & 1.44 & $19.07 * *$ & $10.25^{* *}$ & -0.66 & $5.42 *$ & 2.38 & $1.37 * *$ & $0.87 * *$ & $1.12 * *$ \\
\hline $\mathbf{P}_{2} \times \mathbf{P}_{4}$ & $-1.10^{*}$ & $-1.60 *$ & $-1.35^{*}$ & 6.68 & -0.45 & 3.11 & 4.09 & $4.96^{*}$ & $4.53^{*}$ & $-2.11 * *$ & $-2.21 * *$ & $-2.16^{* *}$ \\
\hline $\mathbf{P}_{2} \times \mathbf{P}_{5}$ & $-2.66^{* *}$ & $-2.42 * *$ & $-2.54 * *$ & 4.78 & -5.79 & -0.51 & 3.04 & -1.02 & 1.01 & $-0.95^{*}$ & $-2.07^{* *}$ & $-1.51^{* *}$ \\
\hline $\mathbf{P}_{2} \times \mathbf{P}_{6}$ & 0.34 & 0.46 & 0.40 & 0.60 & 6.03 & 3.31 & 1.02 & 4.24 & 2.63 & $1.11 * *$ & $0.85^{* *}$ & $0.98^{* *}$ \\
\hline $\mathbf{P}_{2} \times \mathbf{P}_{7}$ & $-2.18 * *$ & $-2.26^{* *}$ & $-2.22 * *$ & 3.12 & $-6.77^{*}$ & -1.83 & 0.64 & -4.54 & -1.95 & $0.79 *$ & $2.25^{* *}$ & $1.52 * *$ \\
\hline $\mathbf{P}_{3} \times \mathbf{P}_{4}$ & $-1.26^{*}$ & $-3.68 * *$ & $-2.47 * *$ & $-12.44 * *$ & $-19.09 * *$ & $-15.77 * *$ & $-5.89 * *$ & $-11.47 * *$ & $-8.68 * *$ & $-0.97 * *$ & $-2.01 * *$ & $-1.49 * *$ \\
\hline $\mathbf{P}_{3} \times \mathbf{P}_{5}$ & -0.52 & 0.40 & -0.06 & $-8.84 *$ & $-12.23 * *$ & $-10.54 * *$ & 0.06 & -0.90 & -0.42 & $-1.81 * *$ & $-3.07 * *$ & $-2.44 * *$ \\
\hline $\mathbf{P}_{3} \times \mathbf{P}_{6}$ & -0.62 & $1.38 *$ & 0.38 & $18.08 * *$ & $9.39 * *$ & $13.73 * *$ & $8.04 * *$ & $8.86 * *$ & $8.45^{* *}$ & $2.25 * *$ & $3.45^{* *}$ & $2.85 * *$ \\
\hline $\mathbf{P}_{3} \times \mathbf{P}_{7}$ & -0.94 & -0.94 & -0.94 & -4.30 & 1.09 & -1.61 & $-4.84 *$ & -1.67 & -3.26 & $0.73^{*}$ & $1.55^{* *}$ & $1.14 * *$ \\
\hline $\mathbf{P}_{4} \times \mathbf{P}_{5}$ & -0.06 & $2.04 * *$ & 0.99 & -1.60 & 4.85 & 1.62 & $6.06^{* *}$ & $5.84 *$ & $5.95 * *$ & $3.31^{* *}$ & $4.15^{* *}$ & $3.73 * *$ \\
\hline $\mathbf{P}_{4} \times \mathbf{P}_{6}$ & $1.54 * *$ & $2.82 * *$ & $2.18 * *$ & 6.52 & $12.67 * *$ & $9.59^{* *}$ & 2.79 & 0.35 & 1.57 & 0.17 & -0.03 & 0.07 \\
\hline $\mathbf{P}_{4} \times \mathbf{P}_{7}$ & -0.08 & 0.80 & 0.36 & -6.16 & -2.43 & -4.30 & $-10.09 * *$ & $-12.18^{* *}$ & $-11.14 * *$ & $0.85^{*}$ & $0.77^{*}$ & $0.81^{*}$ \\
\hline $\mathbf{P}_{5} \times \mathbf{P}_{6}$ & $2.68 * *$ & -1.20 & 0.74 & -6.88 & -2.47 & -4.68 & $-5.76^{* *}$ & -2.93 & -4.34 & $-1.57 * *$ & -0.59 & $-1.08^{* *}$ \\
\hline $\mathbf{P}_{5} \times \mathbf{P}_{7}$ & $2.46^{* *}$ & $3.08 * *$ & $2.77 * *$ & -6.96 & -4.57 & -5.77 & -1.89 & -2.56 & -2.22 & $-1.69 * *$ & $-1.69 * *$ & $-1.69 * *$ \\
\hline $\mathbf{P}_{6} \times \mathbf{P}_{7}$ & $-1.34^{*}$ & $-2.04 * *$ & $-1.69 * *$ & 5.96 & 0.75 & 3.35 & 3.34 & $7.65^{* *}$ & $5.50 *$ & $-1.43 * *$ & $-2.27 * *$ & $-1.85^{* *}$ \\
\hline LSD 5\% $\left(s_{i j}\right)$ & 1.04 & 1.38 & 1.20 & 7.30 & 6.24 & 6.68 & 4.17 & 4.78 & 4.41 & 0.73 & 0.60 & 0.66 \\
\hline LSD 1\% $\left(s_{i j}\right)$ & 1.39 & 1.85 & 1.60 & 9.77 & 8.34 & 8.86 & 5.58 & 6.40 & 5.85 & 0.97 & 0.81 & 0.87 \\
\hline $\operatorname{LSD~5\% }\left(\mathrm{s}_{\mathrm{ij}}-\mathrm{s}_{\mathrm{ik}}\right)$ & 1.61 & 2.14 & 1.87 & 11.31 & 9.66 & 10.35 & 6.46 & 7.41 & 4.84 & 1.13 & 0.93 & 1.02 \\
\hline LSD 1\% $\left(s_{\mathrm{ij}}-\mathrm{S}_{\mathrm{ik}}\right)$ & 2.15 & 2.87 & 2.48 & 15.13 & 12.93 & 13.73 & 8.64 & 9.91 & 6.41 & 1.51 & 1.25 & 1.35 \\
\hline $\operatorname{LSD~5\% }\left(\mathrm{s}_{\mathrm{ij}}-\mathrm{s}_{\mathrm{ki}}\right)$ & 1.39 & 1.86 & 1.62 & 9.80 & 8.37 & 8.97 & 5.59 & 6.42 & 5.92 & 0.98 & 0.81 & 0.88 \\
\hline $\operatorname{LSD} 1 \%\left(\mathrm{~s}_{\mathrm{ij}}-\mathrm{S}_{\mathrm{k} \mathrm{k}}\right)$ & 1.86 & 2.48 & 2.14 & 13.09 & 11.20 & 11.89 & 7.47 & 8.58 & 7.85 & 1.30 & 1.08 & 1.17 \\
\hline
\end{tabular}


Table (5): Cont.

\begin{tabular}{|c|c|c|c|c|c|c|c|c|c|c|c|c|}
\hline \multirow{2}{*}{ Crosses } & \multicolumn{3}{|c|}{ Ear diameter (cm) } & \multicolumn{3}{|c|}{ No. of rows/ear } & \multicolumn{3}{|c|}{ No. of kernels/row } & \multicolumn{3}{|c|}{ Grain yield (ard/fed) } \\
\hline & N1 & $\mathbf{N 2}$ & Comb. & N1 & $\mathbf{N 2}$ & Comb. & N1 & $\mathbf{N 2}$ & Comb. & N1 & $\mathbf{N 2}$ & Comb. \\
\hline $\mathbf{P}_{1} \times \mathbf{P}_{2}$ & -0.02 & 0.17 & 0.08 & -0.23 & -0.07 & -0.15 & 0.06 & -0.89 & -0.42 & 0.62 & $1.94 * *$ & 1.28 \\
\hline $\mathbf{P}_{1} \times \mathbf{P}_{3}$ & 0.08 & -0.11 & -0.01 & 0.35 & 0.23 & 0.29 & $-1.74 * *$ & $-2.49 * *$ & $-2.12^{* *}$ & $4.97 * *$ & $4.66^{* *}$ & $4.82^{* *}$ \\
\hline $\mathbf{P}_{1} \times \mathbf{P}_{4}$ & $0.20^{* *}$ & $0.27 *$ & $0.24 * *$ & $-0.84 * *$ & -0.33 & $-0.59 *$ & $1.28^{*}$ & $2.41 * *$ & $1.84 * *$ & $-2.14 * *$ & 0.94 & -0.60 \\
\hline $\mathbf{P}_{1} \times \mathbf{P}_{5}$ & $0.18^{* *}$ & $0.21 *$ & $0.20^{*}$ & $1.10^{* *}$ & $0.99 * *$ & $1.04 * *$ & 0.26 & 1.35 & 0.80 & -0.33 & $-2.12 * *$ & -1.23 \\
\hline $\mathbf{P}_{1} \times \mathbf{P}_{6}$ & $-0.36^{* *}$ & $-0.33 * *$ & $-0.34 * *$ & 0.35 & -0.37 & -0.01 & $1.44^{*}$ & $2.99 * *$ & $2.21 * *$ & $-2.05^{* *}$ & $-3.68 * *$ & $-2.87 * *$ \\
\hline $\mathbf{P}_{1} \times \mathbf{P}_{7}$ & -0.08 & -0.19 & -0.13 & $-0.73 * *$ & -0.43 & $-0.58^{*}$ & $-1.28^{*}$ & $-3.35^{* *}$ & $-2.32 * *$ & -1.08 & $-1.74 * *$ & $-1.41^{*}$ \\
\hline $\mathbf{P}_{2} \times \mathbf{P}_{3}$ & $-0.28 * *$ & 0.03 & -0.12 & $-0.93 * *$ & 0.17 & -0.38 & 0.48 & $-1.49 *$ & -0.51 & 0.02 & 0.03 & 0.03 \\
\hline $\mathbf{P}_{2} \times \mathbf{P}_{4}$ & 0.04 & -0.09 & -0.02 & -0.12 & $-0.89 * *$ & $-0.51^{*}$ & -1.10 & $-2.59 * *$ & $-1.85^{* *}$ & -1.29 & $-4.32 * *$ & $-2.81^{* *}$ \\
\hline $\mathbf{P}_{2} \times \mathbf{P}_{5}$ & -0.08 & $-0.49 * *$ & $-0.28 * *$ & 0.12 & 0.13 & 0.12 & $-2.62 * *$ & 0.35 & -1.14 & $-2.28 * *$ & -0.46 & $-1.37^{*}$ \\
\hline $\mathbf{P}_{2} \times \mathbf{P}_{6}$ & -0.02 & -0.09 & -0.05 & -0.13 & $-0.93 * *$ & $-0.53 *$ & $-1.94 * *$ & -1.01 & $-1.48^{*}$ & 0.17 & 0.69 & 0.43 \\
\hline $\mathbf{P}_{2} \times \mathbf{P}_{7}$ & $0.36 * *$ & $0.45^{* *}$ & $0.41 * *$ & $1.29 * *$ & $1.61^{* *}$ & $1.45^{* *}$ & $5.14 * *$ & $5.65^{* *}$ & $5.39 * *$ & $2.76^{* *}$ & $2.12 * *$ & $2.44 * *$ \\
\hline $\mathbf{P}_{3} \times \mathbf{P}_{4}$ & -0.06 & -0.21 & -0.14 & 0.16 & -0.09 & 0.03 & 0.02 & 0.51 & 0.26 & $-3.14 * *$ & $-2.52^{* *}$ & $-2.83^{* *}$ \\
\hline $\mathbf{P}_{3} \times \mathbf{P}_{5}$ & 0.02 & -0.07 & -0.02 & -0.30 & $-1.17 * *$ & $-0.74 * *$ & 1.00 & 0.55 & 0.77 & $-4.83^{* *}$ & $-4.35^{* *}$ & $-4.59 * *$ \\
\hline $\mathbf{P}_{3} \times \mathbf{P}_{6}$ & $0.38 * *$ & $0.33 * *$ & $0.36^{* *}$ & $1.15^{* *}$ & $2.47 * *$ & $1.81 * *$ & $2.08 * *$ & $1.89 *$ & $1.98 * *$ & $4.06^{* *}$ & $2.96^{* *}$ & $3.51^{* *}$ \\
\hline $\mathbf{P}_{3} \times \mathbf{P}_{7}$ & $-0.14^{*}$ & -0.03 & -0.08 & $-0.43^{*}$ & $-1.59 * *$ & $-1.01 * *$ & $-1.84^{* *}$ & 1.05 & -0.40 & -1.12 & -0.77 & -0.95 \\
\hline $\mathbf{P}_{4} \times \mathbf{P}_{5}$ & $0.24 * *$ & $0.61 * *$ & $0.43 * *$ & $0.66^{* *}$ & $1.37 * *$ & $1.01 * *$ & $3.72 * *$ & $4.15^{* *}$ & $3.93 * *$ & $5.36^{* *}$ & $4.79 * *$ & $5.07 *$ \\
\hline $\mathbf{P}_{4} \times \mathbf{P}_{6}$ & $-0.40 * *$ & $-0.29 * *$ & $-0.34 * *$ & 0.16 & 0.11 & 0.13 & $-2.60 * *$ & $-2.41 * *$ & $-2.51 * *$ & 0.21 & 0.54 & 0.37 \\
\hline $\mathbf{P}_{4} \times \mathbf{P}_{7}$ & -0.02 & $-0.35^{* *}$ & $-0.18^{*}$ & -0.02 & -0.15 & -0.09 & $-1.32 *$ & $-2.05^{* *}$ & $-1.69 *$ & 1.00 & 0.57 & 0.79 \\
\hline $\mathbf{P}_{5} \times \mathbf{P}_{6}$ & 0.08 & 0.01 & 0.05 & $-1.50 * *$ & $-1.57 * *$ & $-1.54 * *$ & -0.32 & $-3.27 * *$ & $-1.80 * *$ & 0.65 & 0.91 & 0.78 \\
\hline $\mathbf{P}_{5} \times \mathbf{P}_{7}$ & $-0.44 * *$ & -0.25 & $-0.34 * *$ & -0.08 & 0.27 & 0.09 & $-2.04 * *$ & $-3.11 * *$ & $-2.58^{* *}$ & $1.43^{*}$ & $1.28^{*}$ & $1.36^{*}$ \\
\hline $\mathbf{P}_{6} \times \mathbf{P}_{7}$ & $0.32 * *$ & $0.35^{* *}$ & $0.34 * *$ & -0.03 & 0.31 & 0.14 & $1.34^{*}$ & $1.83^{*}$ & $1.58^{*}$ & $-3.03 * *$ & $-1.41^{*}$ & $-2.22 * *$ \\
\hline $\operatorname{LSD~5\% }\left(\mathrm{s}_{\mathrm{ij}}\right)$ & 0.12 & 0.21 & 0.17 & 0.42 & 0.58 & 0.50 & 1.20 & 1.46 & 1.31 & 1.43 & 1.28 & 1.34 \\
\hline LSD 1\% $\left(s_{i j}\right)$ & 0.17 & 0.28 & 0.22 & 0.56 & 0.78 & 0.66 & 1.60 & 1.95 & 1.74 & 1.92 & 1.71 & 1.78 \\
\hline LSD 5\% $\left(s_{\mathrm{ij}}-\mathrm{s}_{\mathrm{ik}}\right)$ & 0.19 & 0.32 & 0.26 & 0.64 & 0.90 & 0.77 & 1.85 & 2.25 & 2.03 & 2.22 & 1.98 & 2.07 \\
\hline LSD 1\% $\left(\mathrm{s}_{\mathrm{ij}} \mathrm{S}_{\mathrm{ik}}\right)$ & 0.26 & 0.43 & 0.35 & 0.86 & 1.21 & 1.02 & 2.48 & 3.02 & 2.69 & 2.97 & 2.66 & 2.75 \\
\hline $\operatorname{LSD} 5 \%\left(\mathbf{s}_{\mathrm{ij}}-\mathbf{S}_{\mathrm{k}}\right)$ & 0.17 & 0.28 & 0.23 & 0.56 & 0.78 & 0.67 & 1.60 & 1.95 & 1.76 & 1.92 & 1.72 & 1.80 \\
\hline LSD 1\% $\left(s_{\mathrm{ij}}-S_{\mathrm{k} 1}\right)$ & 0.22 & 0.37 & 0.30 & 0.75 & 1.04 & 0.89 & 2.14 & 2.61 & 2.33 & 2.57 & 2.30 & 2.38 \\
\hline
\end{tabular}

* and ** significant at 0.05 and 0.01 levels of probability, respectively

\section{REFERENCES}

Abd El-Aty, M. S. M and I. H. I. Darwish (2006). Combining ability and heterosis and their interaction with three nitrogen levels in some yellow maize inbred lines. J Agric Res Tanta Univ., 32(4): 808-830.

Abd El-Aty, M. S., M. A. El-Hity, H. E. Mosa and M. A. A. Hassan (2014). Combining ability analysis in yellow maize under different planting dates and nitrogen rates. Jordan $\mathrm{J}$. Agric. Sci., 10: 237-251.

Abd El-Mottalb, A. A. and H. A. A. Gamea (2013). Combining ability analysis in new white maize inbred lines (Zea mays L.). Minufiya J. Agric. Res., 39(1): 143-151.

Abo El-Haress, S. M. (2015). Diallel analysis for yield, downy mildew and agronomic characters in maize (Zea mays L.). Alex. J. Agric. Res., 60(1): 25-31. 
Adua, G. B, H. Alidua, I. K. Amegbora, M. S. Abdulaia, S. K. Nutsugaha, K. Obeng-Antwib, R. A. L. Kantona, S. S. Buaha, M. J. Kombioka, M. Abudulaia and P. M. Etwirea (2018). Performance of maize populations under different nitrogen rates in northern Ghana. Annals of Agricultural Sci., 63: 145-152.

Ahmed, M. F. (2013). Diallel analysis and biochemical genetic markers for heterosis and combining ability under two sowing dates of maize inbred lines. Asian J. Crop Sci., 5: 81-94.

Al-Naggar, A. M. M., R. Shabana, M. M. M. Atta and T. H. Al-Khalil (2015). Regression of grain yield of maize inbred lines and their diallel crosses on elevated levels of soil-nitrogen. Int. J. Plant Soil Sci., 4: 499-512.

Attia, A. N., M. S. Sultan, M. A. Badawi, M. A. Abdel-Moneam and A. R. M. Al-Rawi (2015). Estimation of combining ability and heterosis for some maize inbred lines and its single crosses. J. Plant Production, Mansoura Univ., 6(1): 83-98.

Badu-Apraku, B. and M. Oyekunle (2012). Genetic analysis of grain yield and other traits of extra-early yellow maize inbreds and hybrid performance under contrasting environments. Field Crops Res., 129: 99-110.

El-Badawy, M. E. M. (2013). Heterosis and combining ability in maize using diallel crosses among seven new inbred lines. Asian J. Crop Sci., 5(1): 1-13.

El-Gonemy, M. A. M. (2015). Combining ability of seven new white maize inbred lines for yield and some agronomic traits. Egypt. J. Plant Breed., 19(1): 15-24.

El-Hosary A. A. A., M. H. Motawea, A. A. Elgammal (2018). Combining ability for yield and some of its attributes in maize across two locations. Egypt. J. Plant Breed., 22(3): 625-640.

El-Hosary, A. A. A. (2014). Comparison between some methods of diallel cross analysis in maize. Egypt. J. Plant Breed., 18(4): 715-736.

El-Shamarka, Sh. A., M. Abdel-Sattar Ahmed and M. M. El-Nahas (2015). Heterosis and combining ability for yield and its components through diallel cross analysis in maize (Zea mays L.). Alex. J. Agric. Res., 60(2): 87-94.

Griffing, B. (1956). Concept of general and specific combining ability in relation to diallel crossing systems. Aus. J. of Biol. Sci., 9: 463-493.

Haque M. M., A. Hamid and N. I. Bhuiyan (2001). Nutrient uptake and productivity as affected by nitrogen and potassium application levels in maize and sweet potato intercropping system. Korean J. Crop Sci., 46(1): 1-5.

Hassan, M. A. A. (2015). Evaluation of new white maize hybrids for resistance to downy mildew and grain yield under different environments. J. Agric. Res. Kafrelsheikh Univ., 41: 565-575.

Kamara, M. M. (2015). Diallel analysis of some yellow maize inbred lines under low and normal nitrogen levels. International $\mathrm{J}$. of Plant Breeding and genetics, 9(2): 32-43.
Kamara, M. M. and M. R. Rehan (2015). Combining ability in maize under two nitrogen levels and assessing genetic diversity using RAPD marker. J. Plant Production, Mansoura Univ., 6(12): 2051-2067.

Kamara, M. M., I. S. El-Degwy and H. Koyama (2014). Estimation combining ability of some maize inbred lines using line $\times$ tester mating design under two nitrogen levels. Aust. J. of Crop Sci., 8(9): 1336-1342.

Katta, Y. S., M. M. Kamara, M. A. El-Hity and H. Koyama (2013). Combining ability for some growthing and yielding traits in maize under two nitrogen levels. Egypt J. Plant Breed., 17(2): 331-345.

Kaur, A., S. Bedi, G. K. Gill and M. Kumar (2012). Effect of nitrogen fertilizers on radiation use efficiency, Crop growth and yield in some maize (Zea mays L.) genotypes. Maydica, 57: 75-82

Makumbi, D., J. F. Betran, M. Bänziger and J. M. Ribaut (2011). Combining ability, heterosis and genetic diversity in tropical maize (Zea mays L.) under stress and non-stress conditions. Euphytica. 180: 143-162.

Medici, L., M. Pereira, P. J. Lea and R. A. Azevedo (2004). Diallel analysis of maize lines with contrasting responses to applied nitrogen. J. Agric. Sci., 142(5): 535-541.

Mosa, H. E. (2003). Heterosis and combining ability in maize (Zea mays L.). Minufiya. J. Agric. Res., 28: 1375-1386.

Mosa, H. E. (2010). Diallel analysis of nine yellow maize inbred lines. Egypt. J. Plant Breed., 14(3): 37-47.

Mosa, H. E., A. A. Motawei and A. M. M. Abd El-Aal (2010). Nitrogen fertilization influence on combining ability for grain yield and resistance to late wilt disease in maize. J. Agric. Res. Kafrelsheikh Univ., 36: 278-291.

Ngaboyisonga, C., K. Njoroge, D. Kirubi and S. M. Githiri (2009). Effects of low nitrogen and drought on genetic parameters of grain yield and endosperm hardness of quality protein maize. Asian J. Agric. Res., 3: 1-10.

Oyekunle, M., B. Badu-Apraku, S. Hearne and J. Franco (2015). Genetic diversity of tropical early maturing maize inbreds and their performance in hybrid combinations under drought and optimum growing conditions. Field Crops Res., 170: 55-65.

San, V. F., A. Bejarano, J. Cross and C. Marin (2001). Heterosis and combining ability of tropical yellow endosperm maize populations. Agronomia tropical, 51(3): 301-318.

Steel, R. G. D. and J. H. Torrie (1980). Principles and Procedures of Statistics. A Biometrical Approach. $2^{\text {nd }}$ Ed. Mc Graw Hill, N.Y., USA.

Wani, M. M. A., S. A. Wani, Z. A. Dar, A. A. Lone, I. Abedi and A. Gazal (2017). Combining ability analysis in early maturing maize inbred lines under temperate conditions, Int. J. Pure App. Biosci., 5(2): 456-466. 


\section{تحليل الهجن التبادلية لبعض سلالات من الذرة الثامية البيضاء تحت مستويين من

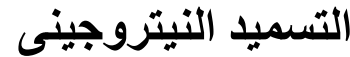

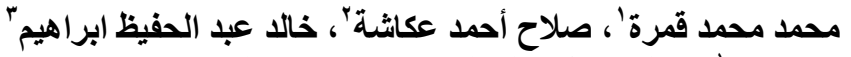

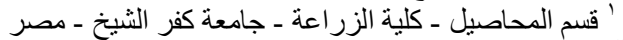

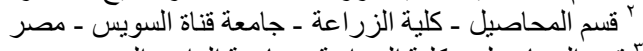

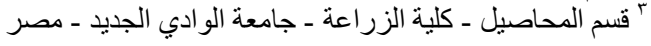

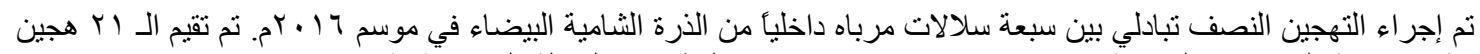

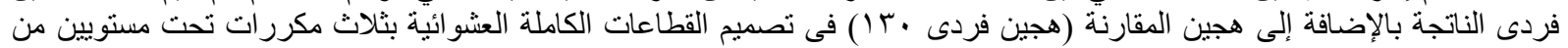

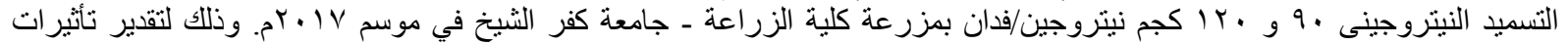

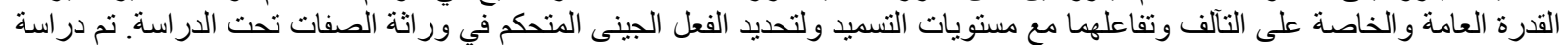

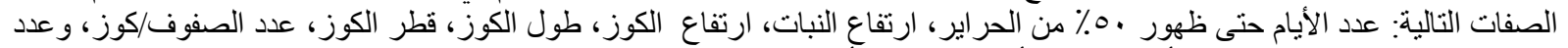

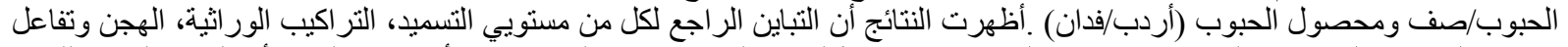

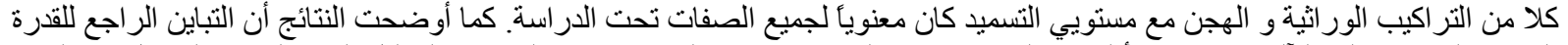

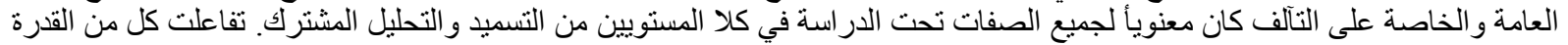

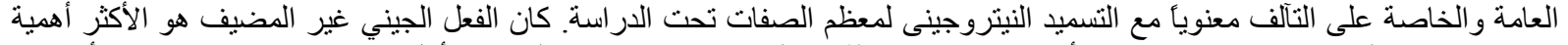

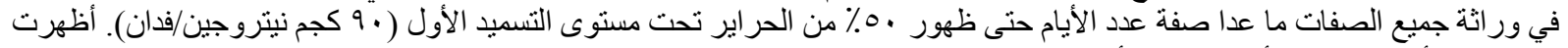

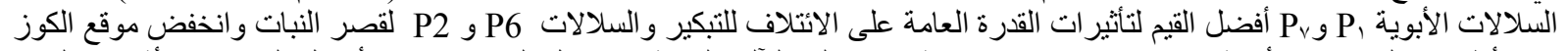

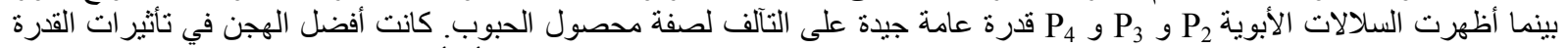
الخاصة على التآلف هي التسميد و التحليل المشترلك بينهم. تفوق محصول الهجينان في كلا المستويين من التسميد و التحليل المشترك بـون بينهما. 